\title{
Negative Norm States in de Sitter Space and QFT without Renormalization Procedure
}

\author{
Mohammad Vahid Takook * \\ October 30, 2018 \\ Department of Physics, Razi University, Kermanshah, IRAN
}

\begin{abstract}
In recent papers [1, 2], it has been shown that the presence of negative norm states or negative frequency solutions are indispensable for a fully covariant quantization of the minimally coupled scalar field in de Sitter space. Their presence, while leaving unchanged the physical content of the theory, offers the advantage of eliminating any ultraviolet divergence in the vacuum energy [2] and infrared divergence in the two point function [3]. We attempt here to extend this method to the interacting quantum field in Minkowski space-time. As an illustration of the procedure, we consider the $\lambda \phi^{4}$ theory in Minkowski space-time. The mathematical consequences of this method is the disappearance of the ultraviolet divergence to the one-loop approximation. This means, the effect of these auxiliary negative norm states is to allow an automatic renormalization of the theory in this approximation.
\end{abstract}

Proposed PACS numbers: 04.62.+v, 03.70+k, 11.10.Cd, 98.80.H

\section{Introduction}

Antoniadis, Iliopoulos and Tomaras [4] have shown that the pathological large-distance behavior (infrared divergence) of the graviton propagator on a de Sitter background does not manifest itself in the quadratic part of the effective action in the one-loop approximation. This means that the pathological behavior of the graviton propagator may be gauge dependent and so should not appear in an effective way as a physical quantity. The linear gravity (the traceless rank-2 "massless" tensor field) on de Sitter space is indeed built up from copies of the minimally coupled scalar field [ [5, 6]. It has been shown [1, 2] that one can construct a covariant quantization of the "massless" minimally coupled scalar field in de Sitter space-time, which is causal and free of any infrared divergence. The essential point of that paper is the unavoidable presence of the negative norm states. Although they do not propagate in the physical space, they play

*e-mail: takook@ccr.jussieu.fr; takook@razi.ac.ir 
a renormalizing role. In the forthcoming paper [6], we shall show that this is also true for linear gravity (the traceless rank-2 "massless" tensor field). These questions have recently been studied by several authors (for minimally coupled scalar field see [5, 7, 2, 3] and for linear gravity see [5, 8, 9, 10]).

The auxiliary states (the negative norm states) appear to be necessary for obtaining a fully covariant quantization of the free minimally coupled scalar field in de Sitter space-time, which is free of any infrared divergence. It has been shown that these auxiliary states automatically renormalize the infrared divergence in the two-point function [3] and removes the ultraviolet divergence in the stress tensor [2]. The crucial point about the minimally coupled scalar field lies in the fact that there is no de Sitter invariant decomposition

$$
\mathcal{H}=\mathcal{H}_{+} \oplus \mathcal{H}_{-}
$$

where $\mathcal{H}_{+}$and $\mathcal{H}_{-}$are Hilbert and anti-Hilbert spaces respectively. For this reason our states contain negative frequency solutions and consequently the use of a Krein space (i.e. Hilbert $\oplus$ anti-Hilbert space) is necessitated. For the scalar massive field where such a decomposition exists as a de Sitter invariant, $\mathcal{H}_{+}$as the usual physical state space $\left(\mathcal{H}_{-}=\mathcal{H}_{+}^{*}\right)$ suffices [2]. It has been also shown that if this method is applied to the free "massive" scalar field in de Sitter space, automatically covariant renormalization of the vacuum energy divergence is obtained [2]. We would like to generalize this method (adding the negative frequency solutions) to the interacting quantum scalar field in Minkowski space-time. These auxiliary states once again, automatically renormalize the problem to the one-loop approximation. In other words, introducing negative frequency solutions plays the key role in the renormalization procedure.

\section{2 de Sitter scalar field}

Let us briefly describe our quantization of the minimally coupled massless scalar field. It is defined by

$$
\square_{H} \phi(x)=0,
$$

where $\square_{H}$ is the Laplace-Beltrami operator on de Sitter space. As proved by Allen [11], the covariant canonical quantization procedure with positive norm states fails in this case. The Allen's result can be reformulated in the following way: the Hilbert space generated by a complete set of modes (named here the positive modes, including the zero mode) is not de Sitter invariant,

$$
\mathcal{H}=\left\{\sum_{k \geq 0} \alpha_{k} \phi_{k} ; \sum_{k \geq 0}\left|\alpha_{k}\right|^{2}<\infty\right\} .
$$

This means that it is not closed under the action of the de Sitter group. Nevertheless, one can obtain a fully covariant quantum field by adopting a new construction [1, 2]. In order to obtain a fully covariant quantum field, we add all the conjugate modes to the previous ones. Consequently, we have to deal with an orthogonal sum of a positive and negative inner product space, which is closed under an indecomposable representation of the de Sitter group. The negative values of the inner product are precisely produced by the conjugate modes: $\left\langle\phi_{k}^{*}, \phi_{k}^{*}\right\rangle=$ $-1, k \geq 0$. We do insist on the fact that the space of solution should contain the unphysical 
states with negative norm. Now, the decomposition of the field operator into positive and negative norm parts reads

$$
\phi(x)=\frac{1}{\sqrt{2}}\left[\phi_{p}(x)+\phi_{n}(x)\right]
$$

where

$$
\phi_{p}(x)=\sum_{k \geq 0} a_{k} \phi_{k}(x)+H . C ., \quad \phi_{n}(x)=\sum_{k \geq 0} b_{k} \phi^{*}(x)+H . C . .
$$

The positive mode $\phi_{p}(x)$ is the scalar field as was used by Allen. The crucial departure from the standard QFT based on CCR lies in the following requirement on commutation relations:

$$
a_{k}\left|0>=0, \quad\left[a_{k}, a_{k^{\prime}}^{\dagger}\right]=\delta_{k k^{\prime}}, \quad b_{k}\right| 0>=0, \quad\left[b_{k}, b_{k^{\prime}}^{\dagger}\right]=-\delta_{k k^{\prime}}
$$

A direct consequence of these formulas is the positivity of the energy i.e.

$$
\left\langle\vec{k}\left|T_{00}\right| \vec{k}\right\rangle \geq 0
$$

for any physical state $|\vec{k}\rangle$ (those built from repeated action of the $a_{k}^{\dagger}$ 's on the vacuum). This quantity vanishes if and only if $|\vec{k}\rangle=|0\rangle$. Therefore the "normal ordering" procedure for eliminating the ultraviolet divergence in the vacuum energy, which appears in the usual QFT is not needed [2]. Another consequence of this formula is a covariant two-point function, which is free of any infrared divergence [3].

This result is the same as that of de Vega et al. [7] where flat coordinate modes solutions were employed. For calculating the Schwinger commutator function, they have not used the twopoint function since in their construction it would result in appearance of a divergence. They calculated the commutator function directly, which resulted in disappearance of the infrared divergence due to the sign of the divergence term. In our alternative method, the Schwinger commutator function was calculated from the finite and covariant two point function [3]. It has been also shown that the Schwinger commutator functions in both methods are one and the same.

\section{Minkowskian free quantum scalar field}

Let us first recall elementary facts about Minkowskian QFT. A classical scalar field $\phi(x)$, which is defined in the 4-dimensional Minkowski space-time, satisfies the field equation

$$
\left(\square+m^{2}\right) \phi(x)=0=\left(\eta^{\mu \nu} \partial_{\mu} \partial_{\nu}+m^{2}\right) \phi(x), \quad \eta^{\mu \nu}=\operatorname{diag}(1,-1,-1,-1) .
$$

Inner or Klein-Gordon product and related norm are defined by 12

$$
\left(\phi_{1}, \phi_{2}\right)=-i \int_{t=\text { cons. }} \phi_{1}(x) \stackrel{\leftrightarrow}{\partial}_{t} \phi_{2}^{*}(x) d^{3} x
$$

Two sets of solutions of (4) are given by:

$$
u_{p}(k, x)=\frac{e^{i \vec{k} \cdot \vec{x}-i w t}}{\sqrt{(2 \pi)^{3} 2 w}}=\frac{e^{-i k . x}}{\sqrt{(2 \pi)^{3} 2 w}}, \quad u_{n}(k, x)=\frac{e^{-i \vec{k} \cdot \vec{x}+i w t}}{\sqrt{(2 \pi)^{3} 2 w}}=\frac{e^{i k . x}}{\sqrt{(2 \pi)^{3} 2 w}}
$$


where $w(\vec{k})=k^{0}=\left(\vec{k} \cdot \vec{k}+m^{2}\right)^{\frac{1}{2}} \geq 0$. These $u(k, x)$ modes are orthogonal and normalized in the sense of $(5)$ :

$$
\begin{gathered}
\left(u_{p}(k, x), u_{p}\left(k^{\prime}, x\right)\right)=\delta^{3}\left(\vec{k}-\vec{k}^{\prime}\right), \\
\left(u_{n}(k, x), u_{n}\left(k^{\prime}, x\right)\right)=-\delta^{3}\left(\vec{k}-\vec{k}^{\prime}\right), \\
\left(u_{p}(k, x), u_{n}\left(k^{\prime}, x\right)\right)=0 .
\end{gathered}
$$

$u_{p}$ modes are positive norm states and the $u_{n}$ 's are negative norm states. The general classical field solution is

$$
\phi(x)=\int d^{3} \vec{k}\left[a(\vec{k}) u_{p}(k, x)+b(\vec{k}) u_{n}(k, x)\right]
$$

where $a(\vec{k})$ and $b(\vec{k})$ are two independent coefficients. The usual quantization of this field is based on the positive norm states only. In the Minkowskian case this choice leads to a covariant quantization (covariant under the proper orthochronous Poincaré group). However, it is well known that an ultraviolet divergence appears in the vacuum energy. This divergence is eliminated with the aid of a "normal ordering" operation.

In the above, we have seen that, in the case of the minimally coupled scalar field in de Sitter space, one cannot construct a covariant quantization of this field with only positive norm states (this fact was proved by Allen in [11]). Also there appears an infrared divergence in the twopoint function built from the positive norm states. For obtaining a covariant quantization and eliminating the infrared divergence the two sets of solutions (positive and negative norms states) are necessary [2]. It has been also shown that the commutator function, which is calculated by these two different methods, is the same [3]. Therefore there exists another possibility for defining the field operator, which satisfies the same commutation relation (locality condition). In contrast to the usual quantization, the field operator acts on the Krein space (positive and negative norms states). Let us show now that, if we use this new method of quantization for the free scalar field in Minkowski space, the ultraviolet divergence in the vacuum energy disappears and there is no need for use the "normal ordering" operation. In Krein QFT the quantum field is defined as follows

$$
\phi(x)=\frac{1}{\sqrt{2}}\left[\phi_{p}(x)+\phi_{n}(x)\right]
$$

where

$$
\begin{aligned}
& \phi_{p}(x)=\int d^{3} \vec{k}\left[a(\vec{k}) u_{p}(k, x)+a^{\dagger}(\vec{k}) u_{p}^{*}(k, x)\right], \\
& \phi_{n}(x)=\int d^{3} \vec{k}\left[b(\vec{k}) u_{n}(k, x)+b^{\dagger}(\vec{k}) u_{n}^{*}(k, x)\right],
\end{aligned}
$$

where $a(\vec{k})$ and $b(\vec{k})$ are two independent operators. The positive mode $\phi_{p}$ is the scalar field as was used in the usual QFT. Creation and annihilation operators are constrained to obey the following commutation rules

$$
\begin{gathered}
{\left[a(\vec{k}), a\left(\vec{k}^{\prime}\right)\right]=0, \quad\left[a^{\dagger}(\vec{k}), a^{\dagger}\left(\vec{k}^{\prime}\right)\right]=0, \quad,\left[a(\vec{k}), a^{\dagger}\left(\vec{k}^{\prime}\right)\right]=\delta\left(\vec{k}-\vec{k}^{\prime}\right),} \\
{\left[b(\vec{k}), b\left(\vec{k}^{\prime}\right)\right]=0, \quad\left[b^{\dagger}(\vec{k}), b^{\dagger}\left(\vec{k}^{\prime}\right)\right]=0, \quad,\left[b(\vec{k}), b^{\dagger}\left(\vec{k}^{\prime}\right)\right]=-\delta\left(\vec{k}-\vec{k}^{\prime}\right),} \\
{\left[a(\vec{k}), b\left(\vec{k}^{\prime}\right)\right]=0, \quad\left[a^{\dagger}(\vec{k}), b^{\dagger}\left(\vec{k}^{\prime}\right)\right]=0, \quad,\left[a(\vec{k}), b^{\dagger}\left(\vec{k}^{\prime}\right)\right]=0, \quad\left[a^{\dagger}(\vec{k}), b\left(\vec{k}^{\prime}\right)\right]=0 .}
\end{gathered}
$$


The vacuum state $\mid 0>$ is then defined by

$$
\begin{gathered}
a^{\dagger}(\vec{k})|0>=| 1_{\vec{k}}>; \quad a(\vec{k}) \mid 0>=0, \forall \vec{k}, \\
b^{\dagger}(\vec{k})|0>=| \overline{1}_{\vec{k}}>; \quad b(\vec{k}) \mid 0>=0, \forall \vec{k}, \\
b(\vec{k})\left|1_{\vec{k}}>=0 ; \quad a(\vec{k})\right| \overline{1}_{\vec{k}}>=0, \forall \vec{k},
\end{gathered}
$$

where $\mid 1_{\vec{k}}>$ is called a one particle state and $\mid \overline{1}_{\vec{k}}>$ is called a one "unparticle state". These commutation relations, together with the normalization of the vacuum

$$
<0 \mid 0>=1
$$

lead to positive (resp. negative) norms on the physical (resp. unphysical) sector:

$$
<1_{\vec{k}^{\prime}}\left|1_{\vec{k}}>=\delta\left(\vec{k}-\vec{k}^{\prime}\right), \quad<\overline{1}_{\vec{k}^{\prime}}\right| \overline{1}_{\vec{k}}>=-\delta\left(\vec{k}-\vec{k}^{\prime}\right) .
$$

If we calculate the energy operator in terms of these Fourier modes, we have

$$
H=\int d^{3} \vec{k} k^{0}\left[a^{\dagger}(\vec{k}) a(\vec{k})+b^{\dagger}(\vec{k}) b(\vec{k})+a^{\dagger}(\vec{k}) b^{\dagger}(\vec{k})+a(\vec{k}) b(\vec{k})\right] .
$$

This energy for the vacuum state is zero and it is not needed to use the "normal ordering" operation. It is also positive for any particles state or physical state $\mid N_{\vec{k}}>$ (those built from repeated action of the $a^{\dagger}(\vec{k})$ 's on the vacuum)

$$
<N_{\vec{k}^{\prime}}|H| N_{\vec{k}^{\prime}}>=\int<N_{\vec{k}^{\prime}}\left|a^{\dagger}(\vec{k}) a(\vec{k})\right| N_{\vec{k}^{\prime}}>k^{0} d^{3} \vec{k} \geq 0, \quad\left|0_{\vec{k}^{\prime}}>\equiv\right| 0>.
$$

We shall attempt to generalize this method to the interacting quantum field in the next section. At this stage we consider various Green's functions fundamental to the interacting case. Within the framework of our approach, the two-point function is the imaginary part the usual Wightman two-point function, which is built from the positive norm states

$$
\mathcal{W}\left(x, x^{\prime}\right)=<0\left|\phi(x) \phi\left(x^{\prime}\right)\right| 0>=\frac{1}{2}\left[\mathcal{W}_{p}\left(x, x^{\prime}\right)+\mathcal{W}_{n}\left(x, x^{\prime}\right)\right]=i \Im \mathcal{W}_{p}\left(x, x^{\prime}\right),
$$

where $\mathcal{W}_{n}=-\mathcal{W}_{p}^{*}$. The commutator and anticommutator of the field are defined respectively by

$$
\begin{gathered}
i G\left(x, x^{\prime}\right)=<0\left|\left[\phi(x), \phi\left(x^{\prime}\right)\right]\right| 0>=2 i \Im \mathcal{W}\left(x, x^{\prime}\right)=2 i \Im \mathcal{W}_{p}\left(x, x^{\prime}\right)=i G_{p}\left(x, x^{\prime}\right), \\
G^{1}\left(x, x^{\prime}\right)=<0\left|\left\{\phi(x), \phi\left(x^{\prime}\right)\right\}\right| 0>=0 .
\end{gathered}
$$

Retarded and advanced Green's functions are defined respectively by

$$
\begin{gathered}
G^{r e t}\left(x, x^{\prime}\right)=-\theta\left(t-t^{\prime}\right) G\left(x, x^{\prime}\right)=G_{p}^{r e t}\left(x, x^{\prime}\right), \\
G^{a d v}\left(x, x^{\prime}\right)=\theta\left(t^{\prime}-t\right) G\left(x, x^{\prime}\right)=G_{p}^{a d v}\left(x, x^{\prime}\right) .
\end{gathered}
$$

The Schwinger commutator function, retarded and advanced Green's functions is the same in the two formalism. The "Feynman" propagator or the Time-ordered product propagator is defined

$$
i G_{T}\left(x, x^{\prime}\right)=<0\left|T \phi(x) \phi\left(x^{\prime}\right)\right| 0>=\theta\left(t-t^{\prime}\right) \mathcal{W}\left(x, x^{\prime}\right)+\theta\left(t^{\prime}-t\right) \mathcal{W}\left(x^{\prime}, x\right) .
$$


In this case we obtain

$$
G_{T}\left(x, x^{\prime}\right)=\frac{1}{2}\left[G_{F}^{p}\left(x, x^{\prime}\right)+\left(G_{F}^{p}\left(x, x^{\prime}\right)\right)^{*}\right]=\Re G_{F}^{p}\left(x, x^{\prime}\right) .
$$

where the positive norm state is

$$
G_{F}^{p}\left(x, x^{\prime}\right)=\int \frac{d^{4} k}{(2 \pi)^{4}} e^{-i k .\left(x-x^{\prime}\right)} \tilde{G}^{p}(k)=\int \frac{d^{4} k}{(2 \pi)^{4}} \frac{e^{-i k .\left(x-x^{\prime}\right)}}{k^{2}-m^{2}+i \epsilon} .
$$

Using the Bessel functions it is also written in the following form

$$
G_{T}\left(x, x^{\prime}\right)=\Re G_{F}^{p}\left(x, x^{\prime}\right)=\frac{m^{2}}{8 \pi} \theta(\sigma) \frac{J_{1}\left(\sqrt{2 m^{2} \sigma}\right)}{\sqrt{2 m^{2} \sigma}}-\frac{1}{8 \pi} \delta(\sigma),
$$

where [12]

$$
\begin{gathered}
G_{F}^{p}\left(x, x^{\prime}\right)=-\frac{1}{8 \pi} \delta(\sigma)+\frac{m^{2}}{8 \pi} \theta(\sigma) \frac{J_{1}\left(\sqrt{2 m^{2} \sigma}\right)-i N_{1}\left(\sqrt{2 m^{2} \sigma}\right)}{\sqrt{2 m^{2} \sigma}} \\
-\frac{i m^{2}}{4 \pi^{2}} \theta(-\sigma) \frac{K_{1}\left(\sqrt{-2 m^{2} \sigma}\right)}{\sqrt{-2 m^{2} \sigma}}, \quad \sigma=\frac{1}{2}\left(x-x^{\prime}\right)^{2} .
\end{gathered}
$$

The ultraviolet singularity appears in the imaginary part of the Feynman propagator $G_{F}^{p}(x, x)$ (equation (9.52) in [12]),

$$
G_{F}^{p}(x, x) \approx \lim _{n \longrightarrow 4} \frac{-2 i}{(4 \pi)^{2}} \frac{m^{2}}{(n-4)}+G_{F}^{\text {finite }}(x, x),
$$

where $G_{F}^{\text {finite }}(x, x)$ is finite as $n \longrightarrow 4$. Then the Green's function $G_{T}$ is convergence in the ultraviolet limit

$$
G_{T}(x, x)=\Re G_{F}^{p}(x, x)=\Re G_{F}^{\text {finite }}(x, x) .
$$

This Green's function for every time-like separated pair $\left(x, x^{\prime}\right)$ is

$$
\lim _{x \rightarrow x^{\prime}} G_{T}\left(x, x^{\prime}\right)=\frac{m^{2}}{16 \pi}
$$

and for space-like separated pair $\left(x, x^{\prime}\right)$ is zero

$$
\lim _{x \rightarrow x^{\prime}} G_{T}\left(x, x^{\prime}\right)=0 .
$$

In the momentum space for this propagator we have [13]

$$
\tilde{G}(k)=\frac{1}{2}\left[\tilde{G}^{p}(k)+\tilde{G}^{p}(k)^{*}\right]=\frac{1}{2}\left[\frac{1}{k^{2}-m^{2}+i \epsilon}+\frac{1}{k^{2}-m^{2}-i \epsilon}\right]=P P \frac{1}{k^{2}-m^{2}},
$$

where $P P$ is the principal part symbol. 


\section{The interaction QFT}

In the interaction case the $\mathrm{S}$ matrix elements, which describes the scattering of the i states into the $\mathrm{f}$ states $\left(S_{f i}=<\right.$ out, $\mathrm{f} \mid$ in, $\left.\mathrm{i}>\right)$ are the most important quantities to be calculated. The $\mathrm{S}$ matrix elements can be written in terms of the time order product of the two free field operator (22) by applying the reduction formulas, Wick's theorem and time evolution operator [13]. As this two point function is convergence in the ultraviolet and infrared limit (26), this method may be renormalized automatically. Here the $\lambda \phi^{4}$ interaction field in Minkowski space is studied to the one-loop approximation.

The tree order S-matrix elements do not change when it is applied to the Krein QFT since the unphysical state disappear in the external line due to the conditions (11) and (14) and the internal propagator in the two cases are the same (28).

In the one-loop approximation case, two primitive divergent integrals appear, which can be written in the following form

$$
\begin{gathered}
-i \Sigma^{p}=\frac{\lambda}{2} \int \frac{d^{4} k}{(2 \pi)^{4}} \frac{1}{k^{2}-m^{2}+i \epsilon}=\frac{\lambda}{2} \lim _{x \rightarrow x^{\prime}} G_{F}^{p}\left(x, x^{\prime} ; m^{2}\right), \\
\Gamma^{4}(s, t, u)=-i \lambda+\Gamma(s)+\Gamma(t)+\Gamma(u),
\end{gathered}
$$

where $s, t$ and $u$ are the Mandelstam variable and

$$
\Gamma(s)=\frac{\lambda^{2}}{2} \int_{0}^{1} d l \int \frac{d^{4} k}{(2 \pi)^{4}} \frac{1}{\left[k^{2}-m^{2}+s l(1-l)+i \epsilon\right]^{2}} .
$$

If we define $M^{2}=m^{2}-s l(1-l)$, this integral can be written in terms of the first one

$$
\begin{aligned}
& \Gamma(s)=\frac{\lambda^{2}}{2} \int_{0}^{1} d l \frac{\partial}{\partial M^{2}} \int \frac{d^{4} k}{(2 \pi)^{4}} \frac{1}{k^{2}-M^{2}+i \epsilon} \\
& =\frac{\lambda^{2}}{2} \int_{0}^{1} d l \frac{\partial}{\partial M^{2}} \lim _{x \rightarrow x^{\prime}} G_{F}^{p}\left(x, x^{\prime} ; M^{2}\right), \quad M^{2} \neq 0 .
\end{aligned}
$$

By using the equation (26) we see simply that if the Green function $G_{F}^{p}$ is replaced by the Green function $G_{T}$ the integrals is convergent.

Now the "self energy" graph is explicitly considered. By using the above discussion (equations (26) and (27)) the self energy terms of the two point function in the new approach is given by

$$
-i \Sigma=\frac{\lambda}{2} \lim _{x \rightarrow x^{\prime}} G_{T}\left(x, x^{\prime} ; m^{2}\right)=\frac{\lambda m^{2}}{32 \pi}, \quad m^{2} \neq 0 .
$$

The positive norm states and the full propagator in the one loop correction are given respectively by

$$
i \Delta(k)=\frac{i}{k^{2}-m^{2}-\frac{\lambda}{32 \pi} m^{2}+i \epsilon}, \quad P P \frac{i}{k^{2}-m^{2}-\frac{\lambda}{32 \pi} m^{2}} .
$$

Then in the one loop correction the mass is replaced by

$$
m^{2}(\lambda)=m^{2}(0)\left[1+\frac{\lambda}{32 \pi}+\ldots . . .\right]
$$


where $m(0)=m$. Due to the interaction, the effective mass of the particle $m(\lambda)$, which determines its response to an externally applied force, is certainly different from the mass of the particle without interaction $m(0)$. In this case, $m(0)$ and $m(\lambda)$ are both measurable.

Finally we calculate explicitly the transition amplitude of the state $\mid q_{1}, q_{2}$; in $>$ to the state $\mid p_{1}, p_{2}$; out $>$ for s-channel contribution in the one-loop approximation. It is given by 13

$$
\begin{gathered}
\mathcal{T} \equiv<p_{1}, p_{2} ; \text { out } \mid q_{1}, q_{2} ; \text { in }>_{s}=\int d^{4} y_{1} d^{4} y_{2} d^{4} x_{1} d^{4} x_{2} e^{i p_{1} . y_{1}+i p_{2} . y_{2}-i q_{1} . x_{1}-i q_{2} . x_{2}} \\
\left(\square_{y_{1}}+m^{2}\right)\left(\square_{y_{2}}+m^{2}\right)\left(\square_{x_{1}}+m^{2}\right)\left(\square_{x_{2}}+m^{2}\right) \frac{(-i \lambda)^{2}}{2 !} \int d^{4} z_{1} d^{4} z_{2}\left[i G_{T}\left(z_{1}-z_{2}\right)\right]^{2} \\
G_{T}\left(y_{1}-z_{2}\right) G_{T}\left(y_{2}-z_{1}\right) G_{T}\left(x_{1}-z_{2}\right) G_{T}\left(x_{2}-z_{2}\right),
\end{gathered}
$$

where the Feynman Green function $G_{F}^{p}$ is replaced by the Time-order product Green function $G_{T}$. We obtain

$$
\begin{gathered}
\mathcal{T}=\frac{\lambda^{2}}{2} \int d^{4} z_{1} d^{4} z_{2} e^{i\left(p_{1}+p_{2}\right) \cdot z_{1}-i\left(q_{1}+q_{2}\right) \cdot z_{2}}\left[G_{T}\left(z_{1}-z_{2}\right)\right]^{2} \\
=\frac{\lambda^{2}}{2}(2 \pi)^{4} \delta^{4}\left(p_{1}+p_{2}-q_{1}-q_{2}\right) \int d^{4} z e^{i\left(p_{1}+p_{2}\right) \cdot z}\left(\frac{m^{2}}{8 \pi} \theta\left(z^{2}\right) \frac{J_{1}\left(\sqrt{m^{2} z^{2}}\right)}{\sqrt{m^{2} z^{2}}}-\frac{1}{4 \pi} \delta\left(z^{2}\right)\right)^{2},
\end{gathered}
$$

where $2 \sigma=\left(z_{1}-z_{2}\right)^{2}=z^{2}$. The integral for the space-like separated pair $\left(z_{1}, z_{2}\right)$ is zero. That means the interaction between the intermediate states do not exist for a space-like separated pair. Then the causality or locality principle is preserved for the intermediate states. By using the equation (26) we obtained that the integral for the light-like separated pair $\left(z_{1}, z_{2}\right)$ is also zero. That means an internal "particle", which propagate in the intermediate states, is "massive" and it can not propagate on the light-cone.

The integral for the time-like separated pair $\left(z_{1}, z_{2}\right)$ is finite

$$
\int d^{4} z e^{i\left(p_{1}+p_{2}\right) \cdot z}\left(\theta\left(z^{2}\right) \frac{J_{1}\left(\sqrt{m^{2} z^{2}}\right)}{\sqrt{m^{2} z^{2}}}\right)^{2}=\text { finite. }
$$

Therefore the transition amplitude is finite in the one-loop approximation.

\section{Conclusion}

We recall that the negative frequency solutions of the field equation are needed for quantizing in a correct way the minimally coupled scalar field in de Sitter space. Contrary to the Minkowski space, the elimination of de Sitter negative norm in the minimally coupled states breaks the de Sitter invariance. Then for restoring the de Sitter invariance, one needs to take into account the negative norm states i.e. the Krein space quantization. It provides a natural tool for renormalization technique [2]. In this paper the $\lambda \phi^{4}$ theory in Minkowski space-time has been studied to the one-loop approximation in the Krein space quantization. It is found that the theory is automatically renormalized in this approximation. The main questions, which naturally arise, are: dose the natural renormalizeability preserve in the higher-loop expansion? does this construction affect the physical world?

Acknowlegements: The author would like to thank S. Rouhani for very useful discussions and S. Teymourpoor and M. Oveisy for their interest in this work. 


\section{References}

[1] S. De Bièvre, J. RenaudJ., Phys. Rev. D, 57(1998)6230

[2] J.P. Gazeau, J. Renaud, M.V. Takook, Class. Quantum Grav., 17(2000)1415, gr-qc/9904023

[3] M.V. Takook, Mod. Phys. Lett. A, 16(2001)1691, gr-qc/0005020

[4] I. Antoniadis, J. Iliopoulos, T.N. Tomaras, Nuclear Phys. B 462(1996)437

[5] M.V. Takook, Thèse de l'université Paris VI, 1997 Théorie quantique des champs pour des systèmes élémentaires "massifs" et de "masse nulle" sur l'espace- temps de de Sitter.

[6] T. Garidi, J. P. Gazeau, J. Renaud, S. Rouhani and M.V. Takook, Linear covariant quantum gravity in de Sitter space, in preparation

[7] H.J. de Vega, J. Ramirez and N. Sanchez, Phys. Rev. D, 60(1999)044007, astro-ph/9812465

[8] M.V. Takook, Proceeding of the Wigsym6, July 16-22 August, 1999, Istanbul, Turkey, grqc/0001052

[9] S.W. Hawking, T. Hertog, N. Turok, Phys. Rev. D, 62(2000)063502; hep-th/0003016

[10] A. Higuchi, S.S. Kouris, gr-qc/0004079, gr-qc/0011062, gr-qc/0107036,

[11] B. Allen, Phys. Rev. D, 32(1985)3136

[12] N.D. Birrell, P.C.W. Davies, Cambridge University Press, (1982) QUANTUM FIELD IN CURVED SPACE

[13] C. Itzykson, J-B. Zuber, McGraw-Hill, Inc. (1988) Quantum Field Theory 